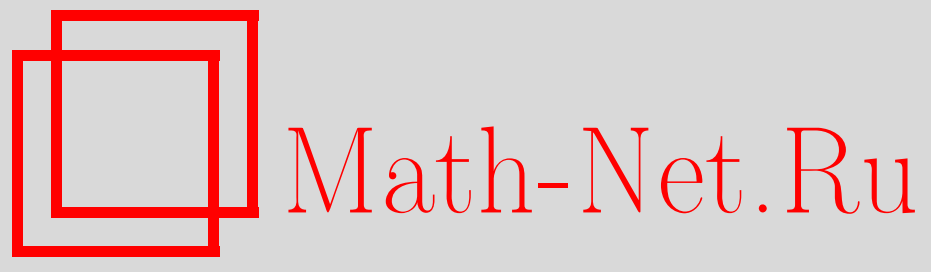

О. И. Рубанов, Хроматические числа трехмерных графов расстояний, не содержащих тетраэдров, Матем. заметки, 2007, том 82, выпуск 5, 797-800

DOI: https://doi.org/10.4213/mzm4091

Использование Общероссийского математического портала Math-Net.Ru подразумевает, что вы прочитали и согласны с пользовательским соглашением http://www . mathnet.ru/rus/agreement

Параметры загрузки:

IP : 54.198 .64 .247

26 апреля 2023 г., $17: 28: 01$

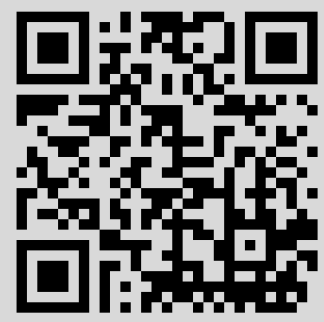




\section{Хроматические числа трехмерных графов расстояний, не содержащих тетраэдров}

\section{О. И. Рубанов}

1. Введение и формулировка результата. Под графом расстояний $G=(V, E)$ мы подразумеваем произвольный граф, множество вершин $V$ которого лежит в метрическом пространстве $(X, \rho)$ (например, в евклидовом пространстве $\left.\left(\mathbb{R}^{n},|\cdot|_{2}\right)\right)$; при этом множество ребер $E$ состоит из всех пар вершин, удаленных друг от друга на определенное расстояние. Одной из классических проблем, связанных с графами расстояний, является проблема нахождения так называемого хроматического числа $\chi((X, \rho), \mathscr{A})$ пространства $(X, \rho)$ с набором запрещенных расстояний $\mathscr{A}$ (см. [1], [2]), которое определяется как минимальное количество цветов, необходимое для такой раскраски точек пространства, что точки, находящиеся на любом расстоянии $a \in \mathscr{A}$, покрашены в разные цвета.

Хорошо известно, что выполнено неравенство $\chi\left(\left(\mathbb{R}^{2},|\cdot|_{2}\right),\{1\}\right) \geqslant 4$, однако проблема получения лучшей нижней оценки или доказательства того, что $\chi\left(\left(\mathbb{R}^{2},|\cdot|_{2}\right),\{1\}\right)=4$, до сих пор остается открытой.

В 1976 г. Эрдеш в [3] сформулировал задачу отыскания графа расстояний без треугольников (или, в более общей постановке, без циклов длины $\leqslant k$ ) с хроматическим числом 4 на плоскости (все существовавшие на тот момент примеры содержали треугольники). Эта задача была решена уже три года спустя Уормалдом в [4], конструкция которого содержала 6448 вершин и не содержала циклов длины 3 (но содержала циклы длины 4). В 1996 г. в [5] О'Доннелл и Хохберг построили граф без 3-циклов на 23 вершинах и граф без 3- и 4-циклов на 45 вершинах.

Естественным обобщением этой задачи является задача построения $n$-мерных графов расстояний, имеющих “большое" хроматическое число и не содержащих клик "большого" размера. Под "большим" хроматическим числом мы подразумеваем хроматическое число, близкое к наилучшим известным нижним оценкам величины $\left.\chi\left(\mathbb{R}^{n},|\cdot| 2\right),\{1\}\right)$. Отметим, что наилучшая нижняя оценка в размерности 3 на сегодняшний день принадлежит Нечуштану: $\chi\left(\left(\mathbb{R}^{3},|\cdot|_{2}\right),\{1\}\right) \geqslant 6$ (см. [6]), а предыдущая оценка $\chi\left(\left(\mathbb{R}^{3},|\cdot|{ }_{2}\right),\{1\}\right) \geqslant 5$ оставалась неулучшенной на протяжении более пятидесяти лет (см. [7]). При $n \rightarrow \infty$ наилучшая оценка имеет вид $\chi\left(\left(\mathbb{R}^{n},|\cdot| 2\right),\{1\}\right) \geqslant(1.239 \ldots+o(1))^{n}$ (см. [8]).

В настоящей работе будет доказана следующая теорема.

Теорема 1. В пространстве $\mathbb{R}^{3}$ существует граф расстояний, имеющий хроматическое число 5 и не содержащий тетраэдров.

Доказательство теоремы абсолютно конструктивно, и мы вкратце изложим его в следующем разделе.

2. Доказательство теоремы 1. Для построения конструкции, которая будет описана ниже, нам потребуется так называемое Мозеровское веретено. Это граф, который состоит из двух пар равносторонних треугольников. Треугольники в каждой паре имеют одну общую сторону, образуя так называемую "иглу". Две "иглы" расположены таким образом, что один "конец" каждой иглы совпадает с "концом" другой, а вторые "концы" удалены на расстояние 1 друг от друга. Мозеровское веретено изображено на рис. 1 . Очевидно, что хроматическое число Мозеровского веретена равно 4.

Приступим непосредственно к построению графа расстояний, удовлетворяющего условию теоремы. Введем в пространстве декартову систему координат, зафиксируем плос-

Работа выполнена при поддержке Российского фонда фундаментальных исследований (грант № 06-01-00383). 


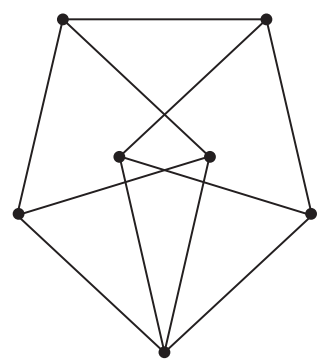

Рис. 1

кость $z=0$. Отметим точку $A$ с координатами $(-1 / 2,0,0)$, точку $B$ с координатами $(1 / 2,0,0)$ и точку $N$ с координатами $(0,1 / 2,0)$. Затем отметим точку $A^{\prime}$, удаленную на расстояние $\sqrt{3} / 2$ от точек $A$ и $N$, и точку $B^{\prime}$, удаленную на расстояние $\sqrt{3} / 2$ от точек $B$ и $N$. И, наконец, отметим на оси ординат точку $T$, симметричную точке $N$ относительно прямой $A^{\prime} B^{\prime}$. Несложно проверить, что

$$
A^{\prime}=\left(-\frac{1}{4}-\frac{\sqrt{5}}{4}, \frac{1}{4}+\frac{\sqrt{5}}{4}, 0\right), \quad B^{\prime}=\left(\frac{1}{4}+\frac{\sqrt{5}}{4}, \frac{1}{4}+\frac{\sqrt{5}}{4}, 0\right), \quad T=\left(0, \frac{\sqrt{5}}{2}, 0\right) .
$$

Далее, построим пары точек $A_{1}$ и $A_{2}, B_{1}$ и $B_{2}$, которые удалены от плоскости $z=0$ на расстояние $1 / 2$ и проекциями которых являются соответственно точки $A^{\prime}$ и $B^{\prime}$. Тогда

$$
\begin{array}{ll}
A_{1}=\left(-\frac{1}{4}-\frac{\sqrt{5}}{4}, \frac{1}{4}+\frac{\sqrt{5}}{4},-\frac{1}{2}\right), & A_{2}=\left(-\frac{1}{4}-\frac{\sqrt{5}}{4}, \frac{1}{4}+\frac{\sqrt{5}}{4}, \frac{1}{2}\right), \\
B_{1}=\left(\frac{1}{4}+\frac{\sqrt{5}}{4}, \frac{1}{4}+\frac{\sqrt{5}}{4},-\frac{1}{2}\right), & B_{2}=\left(\frac{1}{4}+\frac{\sqrt{5}}{4}, \frac{1}{4}+\frac{\sqrt{5}}{4}, \frac{1}{2}\right) .
\end{array}
$$

Заметим, что точки $A_{1}$ и $A_{2}$ удалены от точек $A, T$ и $N$ на расстояние 1 , а точки $B_{1}$ и $B_{2}$ удалены от точек $B, T$ и $N$ на расстояние 1 .

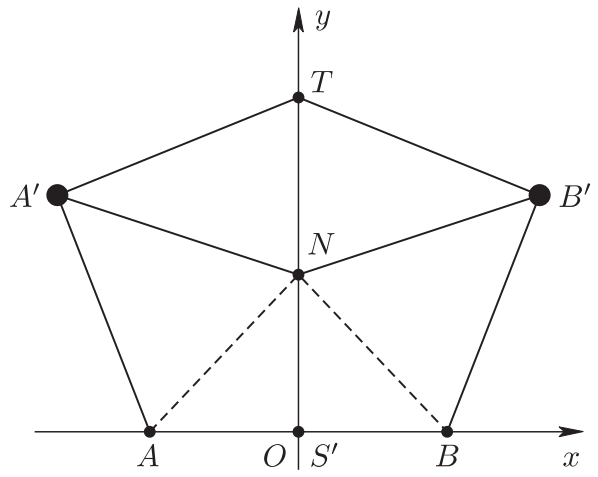

Рис. 2

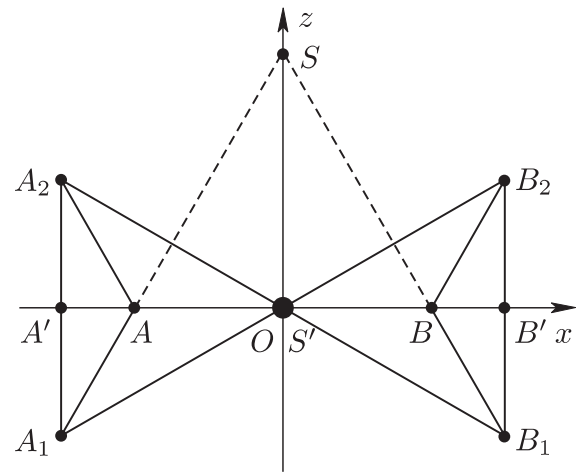

Рис. 3

Кроме того, построим точку $S$, удаленную от точек $A, B$ и $N$ на расстояние 1 . Без ограничения общности можем считать, что $S=(0,0, \sqrt{3} / 2)$. Пусть $S^{\prime}=(0,0,0)$ - это проекция точки $S$ на плоскость $z=0$. Отметим также, что $|S T|=\sqrt{2}\left(|\cdot|=|\cdot|_{2}\right)$. На рис. 2 и 3 изображены проекции описанного графа на плоскости $z=0$ и $y=0$. 
Заметим, что вершины $A, B, A_{1}, A_{2}, B_{1}, B_{2}$ и $N$ образуют Мозеровское веретено (две "иглы": $A, A_{1}, A_{2}, N$ и $\left.B, B_{1}, B_{2}, N ;|A B|=1\right)$. Также отметим, что точка $S$ удалена на расстояние 1 от точек $A, B$ и $N$, а точка $T$ удалена на расстояние 1 от точек $A_{1}, A_{2}, B_{1}$ и $B_{2}$. Таким образом, каждая точка Мозеровского веретена соединена ребром либо с $S$, либо с $T$.

Перейдем к завершающему этапу построения. Вначале построим правильную четырехугольную пирамиду со стороной 1 . Она изображена на рис. 4.

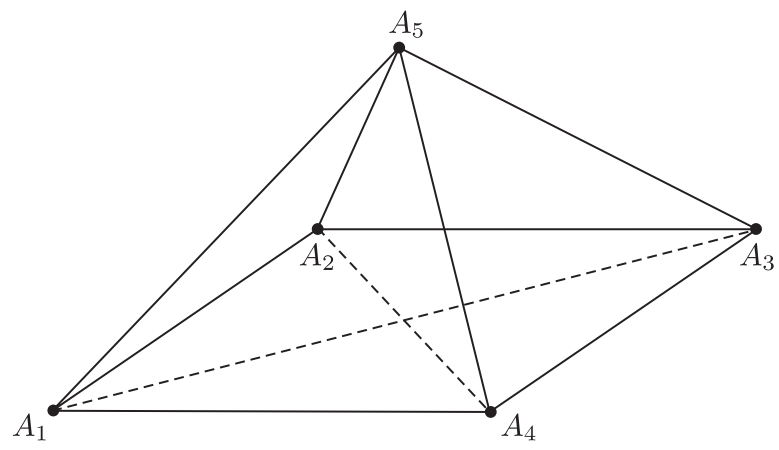

Рис. 4

До полного графа на пяти вершинах четырехугольной пирамиде "не достает" двух ребер $\left(A_{1} A_{3}\right.$ и $\left.A_{2} A_{4}\right)$. Построим на парах вершин $A_{1}, A_{3}$ и $A_{2}, A_{4}$ описанную выше конструкцию. Один экземпляр этой конструкции будет построен на вершинах $A_{1}$ и $A_{3}$, и этим вершинам будут соответствовать вершины $S$ и $T$ конструкции. Второй экземпляр мы построим, соответственно, на вершинах $A_{2}$ и $A_{4}$. Это построение возможно, поскольку $\left|A_{1} A_{3}\right|=\left|A_{2} A_{4}\right|=|S T|=\sqrt{2}$. Теперь искомый граф расстояний $G$ окончательно построен, осталось лишь доказать, что его хроматическое число не меньше пяти и что он не содержит тетраэдров.

Докажем вначале, что хроматическое число построенного графа не меньше пяти. Действительно, предположим, что нам удалось покрасить вершины графа в четыре цвета. Тогда, очевидно, что хотя бы в одной из пар вершин $A_{1}, A_{3}$ и $A_{2}, A_{4}$ обе вершины имеют один и тот же цвет (скажем, “красный”). Пусть, без ограничения общности, это пара вершин $A_{1}, A_{3}$. Рассмотрим теперь Мозеровское веретено, которое соответствовало этой паре одноцветных вершин. Заметим, что каждая вершина этого веретена соединена ребром с одной из вершин $A_{1}, A_{3}$, поэтому ни одна вершина веретена не может быть покрашена в красный цвет. Таким образом, Мозеровское веретено покрашено в три оставшихся цвета. Но, как мы знаем, хроматическое число Мозеровского веретена равно четырем. Противоречие. Значит, хроматическое число графа $G$ не меньше пяти.

Осталось отметить, что этот граф не содержит тетраэдров. На доказательстве этого факта мы подробно останавливаться не будем, скажем лишь, что отсутствие в графе $G$ тетраэдров достигается вращением добавляемых Мозеровских веретен относительно осей, проходящих через пары точек $A_{1}, A_{3}$ и $A_{2}, A_{4}$, и небольшим перебором.

Теорема доказана. Добавим, что полученный граф имеет 19 вершин и 44 ребра.

3. Несколько дополнительных замечаний. Следуя приведенному выше доказательству, мы можем доказать еще одну теорему.

Теорема 2. В пространстве $\mathbb{R}^{n}$ существует граф расстояний, имеющий хроматическое число $n+2$ и не содержащий $n$-мерных симплексов. 
В настоящей краткой заметке мы не приводим доказательство этой теоремы. Заметим лишь, что конструкция будет аналогична той, которая была построена в предыдущем разделе, но вместо Мозеровского веретена будет использоваться его $(n-1)$-мерный аналог - граф Райского (см. [7]).

Применив вероятностный метод, можно обнаружить, что при $n \rightarrow \infty$ существуют графы расстояний, имеющие экспоненциально большое хроматическое число и не содержащие симплексов размерности $\geqslant \varphi(n)$ с некоторым $\varphi(n)=o(n)$. В определенном смысле, это более сильный результат, однако построенный в теореме 2 пример имеет ряд преимуществ: он полностью конструктивен, содержит малое число вершин (а именно, $5 n+4)$ и может давать уточнение вероятностного результата при малых $n$.

\section{СПИСОК ЦИТИРОВАННОЙ ЛИТЕРАТУРЫ}

[1] А. М. Райгородский, УМH, 56:1 (2001), 107-146. [2] A. Soifer, Geombinatorics, 1:3 (1991), 13-15. [3] P. Erdős, Unsolved Problems, Proc. Fifth Brit. Comb. Conf. (Univ. Aberdeen, Aberdeen, 1975), Util. Math. Publ., Winnipeg, 1976. [4] N. Wormald, J. Austral. Math. Soc. Ser. A, 28:1 (1979), 1-8. [5] R. Hochberg, P. O'Donnel, Geombinatorics, 5:4 (1996), 137-141. [6] О. Nechushtan, Discrete Math., 256:1-2 (2002), 499-507. [7] Д. Е. Райский, Матем. заметки, 7 (1970), 319-323. [8] А. М. Райгородский, УМН, 55:2 (2000), 147-148.

\section{О. И. Рубанов}

Поступило

Московский государственный

14.05.2007

университет им. М. В. Ломоносова

E-mail: leg@pisem.net 